\title{
Automatic vs. Manual Multi-Display Configuration
}

\author{
A Study of User Performance in a Semi-Cooperative Task Setting
}

\author{
Thomas Heider, Thomas Kirste \\ Institute of Computer Science \\ Rostock University, Germany \\ $\{$ th,tk\}@informatik.uni-rostock.de
}

\begin{abstract}
Emerging multi-display infrastructures provide users with a large number of (semi-) public and private displays. Selecting what information to present on which display here becomes a real issue, especially when multiple users with diverging interests have to be considered. This especially holds for dynamic ensembles of displays. Therefore, automatic assignment strategies might be useful, if they are able to provide the required assignment precision. We claim that it is possible to define such strategies, and show that it is able to assist users in solving specific tasks in multi-display environments at least as effectively as conventional manual assignment. Our claims are based on user performance data collected in the scope of a comparison study.
\end{abstract}

\section{Categories and Subject Descriptors}

H.5.2 [Information Interfaces and Presentation]: User Interfaces-Evaluation/methodology

\section{Keywords}

Multi-Display Environments, Pro-active Meeting Support

\section{MULTI-DISPLAY ENVIRONMENTS}

Multi-Display Environments (see e.g. the UbiComp 2004 workshop on Ubiquitous Display Environments [1] or the Ubicomp 2006 workshop on next generation conference rooms [2]) support collaborative problem solving and teamwork by providing multiple display surfaces for presenting information. Typical examples for such environments are meeting rooms, conference rooms, and "mission control centers".

One difficult task here is the Display Mapping problem that is, deciding which information to present on what display in order to optimally satisfy the users' needs for information. While this task is more or less trivial in single-user, single-display situations, it becomes challenging in multiuser, multi-display settings: Users and displays are spatially dispersed so that the visibility of (semi-) public and private

(C) Thomas Heider, Thomas Kirste, 2007 Published by the British Computer Society Volume 2 Proceedings of the 21st BCS HCI Group Conference

HCI 2007, 3-7 September 2007, Lancaster University, UK Devina Ramduny-Ellis \& Dorothy Rachovides (Editors) displays varies across users. Also, information needs may vary across users, so that finding the "best" assignment of information to displays becomes a difficult problem.

Current approaches for controlling multi-display environments rely on manual assignment, using a suitable interactive interface, resolving conflicts by social protocols (negotiations). One example is the ModSlideShow system [3], which is designed to manage presentation slides on multiple displays. For assignment of content to displays, meeting participants drag-and-drop presentations from their note books to any of the available displays. Another similar system is the PointRight software developed for Stanford's Meyer Teamspace [4]. However, manual display assignment has to cope with the following problems:

- Interest conflicts between users might be solved faster by computer supported negotiation mechanism: Morris et al. [5] have already observed that social protocols do not always suffice for coordinating the use of shared resources, such as display surfaces, in teams - even in relatively simple situations. They suspect that the need for coordination may increase as the number of users, the number of documents, or the number or size of the surfaces increases. Indeed, they advocate the development of specific strategies for automatizing the negotiation process.

- The need for dynamic realignment of Display Mapping is caused by topic changes in the user population - in this situation, the user's focus of attention will be on the changing topic rather than on convincing the display infrastructure to change the topic.

Therefore, an automatic display assignment might be helpful in multi-display environments, specifically in multi-user settings. However, to our knowledge, it is not known if suitable automatic assignment heuristics can be found. This is the question we want to answer.

\section{DISPLAY MAPPING QUALITY}

A display mapping is a function $m$, which assigns documents to sets of displays. For a given document $d, m(d)$ gives the set of displays document $d$ is assigned to. (It sometimes clearly makes sense to assign a document to more than one display.)

In order for automatic display mapping to be successful it is necessary to identify a well-defined quality measure that 


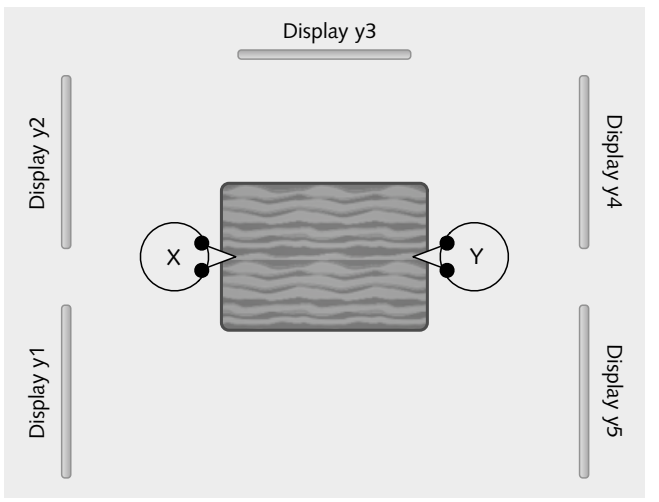

Figure 1: Experimental setup

sufficiently captures the users needs. Clearly, at least the following aspects seem reasonable:

Spatial Layout: For documents of high importance to a user, displays should be preferred that provide a good visibility for the user. Formally, this critierion $m$ can be defined as

$$
q_{s}(m)=\sum_{\substack{u \in U \\ d \in D}} \operatorname{impt}(d, u) * \max _{y \in m(d)} \operatorname{vis}(y, u)
$$

where $\operatorname{impt}(d, u) \in[0 \ldots 1]$ denotes the importance of the document $d$ to a user $u$, and $\operatorname{vis}(y, u) \in[0 \ldots 1]$ the visibility of display $y$ by user $u$. If a document is assigned to multiple displays, only the best one for a given user is considered when computing the quality for this user (this is the "max vis" term). We call this display "primary display".

Deriving a reliable estimation of impt in general may be a substantial challenge - however, there may be additional informations available that can be used as a surrogate (such as an agenda item listing a responsible person with a number of associated documents, etc.). In our test, we have used a manual importance assigment.

Temporal Continuity: When considering a display for a document, the system should prefer already existing assignments: Documents should not unnecessarily change their place. A relevant display shift occurs between two mappings, if a user's primary display for a document changes. We then try to minimize these shifts relative to the document's importance.

Based on these criteria, we have developed an algorithm that is able to automatically compute a display mapping for a set of users and documents (see [6] for more details).

\section{EVALUATION \\ 3.1 Experimental Design}

The objective of our evaluation experiment was to compare the effect of manual and automatic display assignment on task performance. Our hypotheses is that automatic assignment, even using the straightforward quality measure outlined above, is able to provide the same quality as a manual assignment.
Specifically, we have measured the impact of manual vs. automatic display assignment on the performance of a team in solving a semi-cooperative task. In such tasks, the need of cooperation and joint use of information is not evident from the start, but rather arises while working on the task. We think that this kind of aspect pertains to many team processes, specifically in multidisciplinary teams.

Setup. Two-person teams had to solve a semi-cooperative set of comparison tasks as fast as possible. The two team members, $\mathrm{X}$ and $\mathrm{Y}$, were given different agendas, each containing the description of an individual comparison. For $\mathrm{X}$ the task was to compare two documents $\mathrm{A}$ and $\mathrm{B}$, for $\mathrm{Y}$ the task was to compare A and C. The task was a simple letter comparison, counting the number of differences in the two letter sequences contained in A and B resp. A and C. In addition, $\mathrm{X}$ and $\mathrm{Y}$ had to report time information and a random key from another document Time. The seemingly unrelated tasks for $\mathrm{X}$ and $\mathrm{Y}$ were linked into a cooperative task through the shared documents A and Time.

The experimental environment provided five projection based public displays (see Figure 1). Note that there are two pairs of displays exclusively visible to $\mathrm{X}$ and $\mathrm{Y}$, respectively, and one display visible to both $\mathrm{X}$ and $\mathrm{Y}$. For the experiments, every participant was given a simple user interface for document assignment. Manually assignment of a document to a display-surface is done through a simple "drag \& drop". For automatic assignment, the user just associates an importance value with the documents.

As the agendas and task descriptions were mutually unknown, the sharing had to be discovered through a conflict in the manual assignment group. (In order to enforce resource conflicts in this simple setting, each document could only be displayed on one display at a time.)

For each experiment, we recorded the time required for completing the task, the number of interactions, and the solution correctness (percentage of letter differences found). After each task set, the subjects were asked to answer a questionnaire regarding user satisfaction. After both task sets, the subjects were asked to complete a final questionnaire regarding the comparison of the automatic and the manual assignment.

Participants. 24 voluntary subjects (19 male and 5 female) were recruited from staff members and students of the local university. The participants were between the ages of 20 and 41 and were used to computer systems. The participants were randomly grouped into 12 teams, 6 in group A and 6 in group M. The teams had to solve two sets of comparison tasks in sequence. Group A had to solve the first set using automatic assignment and the second set with manual assignment. The Group $M$ was given the tasks in reverse order. In the evaluation of the results, we will call the first set "Initial Test" and the second "After Training", respectively.

Summary of experimental design

Group A:
\begin{tabular}{|c|c|c|c|}
\hline $\begin{array}{c}\text { First } \\
\text { Task Set } \\
\text { (Initial Test) }\end{array}$ & $\begin{array}{c}\text { Second } \\
\text { Task Set } \\
\text { (After Training) }\end{array}$ & $\begin{array}{c}\text { First } \\
\text { Task Set } \\
\text { (Initial Test) }\end{array}$ & $\begin{array}{c}\text { Second } \\
\text { Task Set } \\
\text { (After Training) }\end{array}$ \\
\hline Automatic & Manual & Manual & Automatic \\
\hline
\end{tabular}


Solution Time

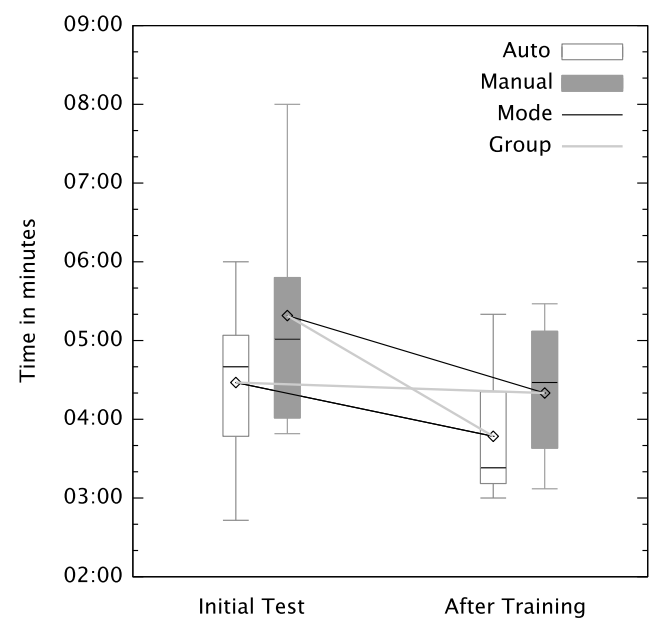

Interactions

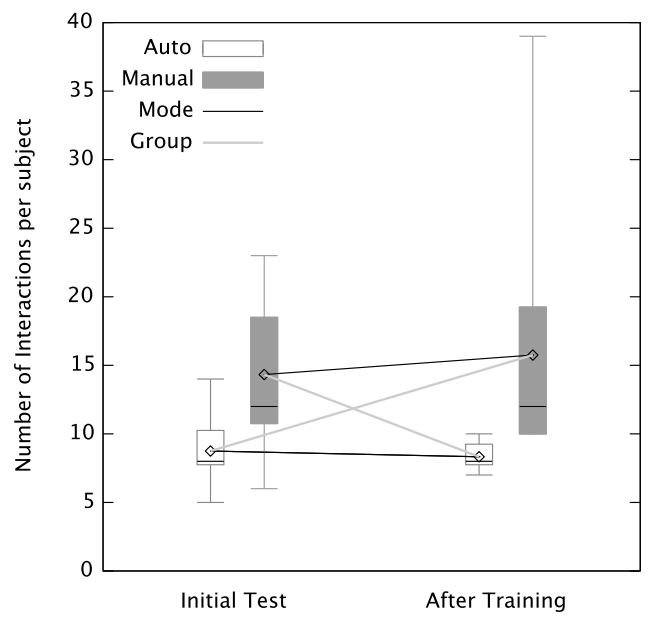

Figure 2: Boxplots of solution time vs. mode, overall (left) and interaction count vs. mode, (right)

Note. A goal of the experimental design has been to (I) explicitly provoke conflicts between the team members regarding the use of the available display space and (II) to enforce substantial changes in the set of documents currently important for a user. Clearly, display assignment becomes an issue only, once more relevant documents than displays are available (specifically, if different users have different sets of relevant documents), and once the set of currently relevant documents changes dynamically.

\subsection{Results}

Performance. On average all subjects needed 4:28 min to complete one set of a comparison task. When the teams were using automatic assignment, the average time was 4:08 min, while they required an average time of 4:49 min using manual assignment. The overall average number of interactions was 11.8 , where the subjects needed 8.5 interactions on average with automatic and 15 interactions on average with manual assignment. The average solution correctness was $95 \%$, for both manual and automatic assignment.

This indicates that the automatic assignment is superior to manual assignment, regarding time and interactions (a brief statistical validation for this claim is given further below).

An overview of the collected data is shown in the boxplots in Figure 2 and $3^{1}$. In these plots, "mode" refers to the display assignment mode (manual vs. automatic). In the per-task-set plots, grey lines connect the mean values of the two consecutive task sets of a group (Group A or Group $\mathrm{M})$, black lines connect consecutive task sets using the same assignment mode.

As can be seen in Figure 2, left, for both task sets the solution time is shorter when using automatic assignment. In addition, Group $M$ was able to solve the task substantially faster in the second set (i.e., when switching from manual to

${ }^{1}$ These boxplots show the minimum and maximum values, the $25 \%$ and $75 \%$ percentiles, the median (horizontal bar inside the box), and the mean (small circle inside the box). automatic assignment), whereby Group A was not able to improve performance in the second set (i.e., switching from automatic to manual assignment). The number of interactions (Figure 2, right) is smaller for the automatic method in both sets. Interestingly, the interaction counts within a mode are almost identical in both sets. There was no training effect. This indicates, that the training (due to solving similar task in both sets) had no influence in using the system infrastructure. The training effect was limited to solving the key problem of comparing the letter sequences.

In the manual assignment mode, both groups initially had no idea that they needed to share documents. So they unwittingly "stole" the shared documents from each others "private" displays. It took a couple of interactions until the participants realized that they needed to cooperate and to assign some of the documents to a display visible to both users. This process of realization and negotiation was the reason for confusion and delay (manifesting itself in the higher solution time and interaction counts required in the manual mode). Interestingly, even the Group A did not realize that they had to share documents in the manual task set (second task set for Group A), although they might have been able to discover this fact in the first task set.

In the automatic assignment mode no such conflicts did arise as the system automatically displayed shared documents on a shared screen. If we use the number of interactions as indicator of occurred conflicts, the data shows that with the automatic mode the number of conflicts is considerably smaller than in the manual mode. A detailed survey of the $\log$ files showed that documents which had to be shared, very frequently were reassigned in the manual mode. This proves the presumption that resolving conflicts by social negotiation is - in some situations - inferior to a computer supported negotiation, which can be solved by an automatic assignment using a global quality function such as $q$.

User Satisfaction. For assessing user satisfaction, we used parts of the technology acceptance model (TAM). We in- 


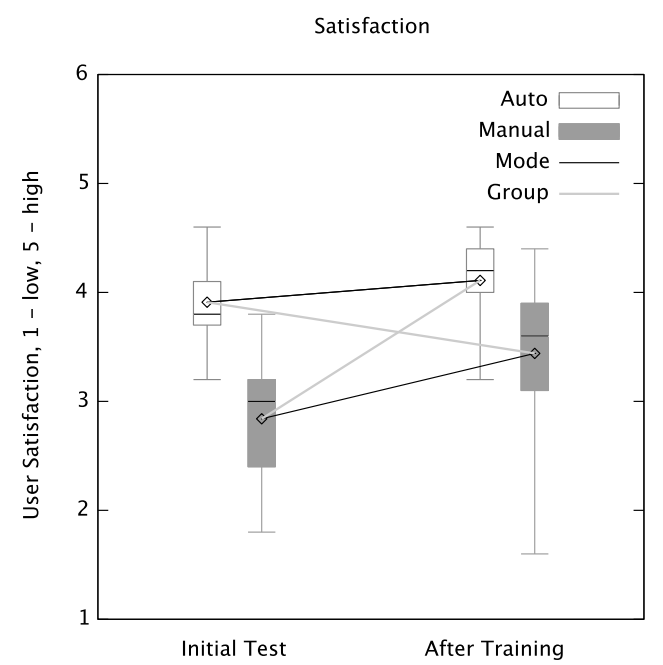

Figure 3: Boxplots of user satisfaction vs. mode

cluded the following items, each to be answered on a scale from 1 (strongly disagree) to 5 (strongly agree):

- The system is easy to use.

- The system helps in solving the task efficiently.

- It is easy to cooperate with the team partner.

- The system helps in solving team conflicts.

- I felt comfortable in using the system.

The final questionnaire used the same items, but with the request to compare both approaches, automatic and manual assignment, on a scale from 1 (manual assignment strongly preferred) to 5 (automatic assignment strongly preferred).

The distribution of the user satisfaction data (using perquestionaire averages) is shown in Figure 3. The overall user satisfaction is higher in the auto mode, for both task sets. In addition, user satisfaction decreases within a group when switching from auto to manual, while it increases when switching from manual to auto.

The correlation of the subjective user satisfaction with the objective data from the log files confirm our hypothesis that the automatic display assignment is superior to the manual assignment in multi-user, multi-display situations with conflicting and dynamic document sets.

For assessing the statistical validity of the results for solution time $t$, interaction count $i$, and overall satisfaction $s$, we have used a one-sided $t$-test (assuming unknown and not necessarily equal variances for the automatic and the manual test results). The null hypothesis in each case has been that the manual method is at least as good as the automatic method. The alternative hypothesis in each case is that automatic assignment is superior to manual assignment. As can bee seen from the results below, the null hypothesis can be rejected in all cases. Therefore we conclude that automatic assignment for multi-user and multi-display situations is superior to manual assignment.

\begin{tabular}{c|c|c}
$H_{0}$ & $H_{1}$ & $H_{0}$ rejected at level \\
\hline$t_{\text {man }} \leq t_{\text {auto }}$ & $t_{\text {man }}>t_{\text {auto }}$ & $2.5 \%$ \\
\hline$i_{\text {man }} \leq i_{\text {auto }}$ & $i_{\text {man }}>i_{\text {auto }}$ & $0.5 \%$ \\
\hline$s_{\text {man }} \geq s_{\text {auto }}$ & $s_{\text {man }}<s_{\text {auto }}$ & $0.5 \%$ \\
\hline
\end{tabular}

\section{SUMMARY}

We have discussed the problem of assisting teams in effectively using multi-display environments for working together and we have addressed the question whether it is possible to find well-defined quality criteria for automatic display assignment.

We have proposed two criteria (spatial quality and temporal continuity). Our user studies show that - at least for specific scenarios - an automatic display assignment based on these heuristics can be at least as good as a manual assignment. Therefore, it proves that it is possible to successfully identify a set of quality criteria for automatic display assignment.

Indeed, we have even been able to show that automatic assignment enables teams to solve their tasks in a shorter time, with less conflicts between team members, and with greater satisfaction.

\section{REFERENCES}

[1] Alois Ferscha, Gerd Kortuem, and Antonio Krüger. Workshop on ubiquitous display environments. In Proc. Ubicomp 2004, Nottingham, England, Sep 72004.

[2] Maribeth Back et al. Workshop on ubiquitous display environmentssable ubiquitous computing in next generation conference rooms: design, architecture and evaluation. In Ubicomp 2006, Newport Beach, CA, USA, Sep 172006.

[3] Patrick Chiu, Qiong Liu, John S. Boreczky, Jonathan Foote, Don Kimber, Surapong Lertsithichai, and Chunyuan Liao. Manipulating and annotating slides in a multi-display environment. In Human-Computer Interaction INTERACT '03: IFIP TC13 International Conference on Human-Computer Interaction, 2003.

[4] Brad Johanson, Greg Hutchins, Terry Winograd, and Maureen Stone. Pointright: Experience with flexible input redirection in interactive workspaces. In Proc. ACM Conference on User Interface and Software Technology (UIST2002), pages 227-234, Paris, France, 2002.

[5] Meredith R. Morris, Kathy Ryall, Chia Shen, Clifton Forlines, and Frederic Vernier. Beyond "social protocols": multi-user coordination policies for co-located groupware. In $C S C W$ '04: Proceedings of the 2004 ACM conference on Computer supported cooperative work, pages 262-265, New York, NY, USA, 2004. ACM Press.

[6] Thomas Heider, Martin Giersich, and Thomas Kirste. Resource optimization in multi-display environments with distributed grasp. In Proceedings of the First International Conference on Ambient Intelligence Developments (AmI.d'06), pages $60-76$, Sophia Antipolis, France, September 19 - 22 2006. Springer. 